\title{
Decision making for patients with multiple brain metastases: radiosurgery, radiotherapy, or resection?
}

\author{
Douglas Kondziolka, M.D., M.Sc., F.R.C.S.(C), Atul Patel, M.D., \\ L. DAdE LunSFORD, M.D., AND John C. FLICKINGER, M.D.
}

\begin{abstract}
Departments of Neurological Surgery and Radiation Oncology, and the Center for Image-Guided Neurosurgery, University of Pittsburgh, Pittsburgh, Pennsylvania
\end{abstract}

\begin{abstract}
Object. Multiple brain metastases are a common health problem, frequently found in patients with cancer. The prognosis, even after treatment with whole-brain radiation therapy (WBRT), is poor, with an average expected survival time of less than 6 months. Investigators at numerous centers have evaluated the role of stereotactic radiosurgery in retrospective case series of patients harboring solitary or multiple tumors. Tumor resection is used mainly for patients with large tumors that cause acute neurological syndromes. The authors conducted a randomized trial in which they compared radiosurgery combined with WBRT with WBRT alone.

Methods. Twenty-seven patients were randomized (14 to recieve WBRT alone and 13 to receive WBRT combined with radiosurgery). The rate of local failure at 1 year was $100 \%$ after WBRT alone but only $8 \%$ in patients in whom boost radiosurgery was performed. The median time to local failure was 6 months after WBRT alone (95\% confidence interval (CI) 3.5-8.5) in comparison to 36 months $(95 \%$ CI 15.6-57) after WBRT and radiosurgery $(\mathrm{p}=0.0005)$. The median time to the development of any brain failure was improved in the combined modality group $(\mathrm{p}=0.002)$. Survival was shown to be related to the extent of extracranial disease $(p=0.02)$.

Conclusions. Combined WBRT and radiosurgery for the treatment of patients with two to four brain metastases significantly improves control of brain disease. Whole-brain radiation therapy alone does not provide lasting and effective care when treating most patients. Surgical resection remains important for patients with large symptomatic tumors and in whom limited extracranial disease has been demonstrated.
\end{abstract}

KEY WoRDS - multiple brain metastases - cancer • craniotomy - radiation therapy • radiosurgery • survival

Brain metastases develop in up to $50 \%$ of all patients with cancer. $^{25}$ In most series, the use of fractionated WBRT extends a patient's survival by 3 to 5 months., ${ }^{5,7,8,23}$ Although more patients harbor multiple rather than solitary brain metastases at presentation (over 100,000 patients with solitary tumors are diagnosed in the United States each year), few gains have been made in the treatment of this disease. For patients with a single brain metastasis, the authors of two randomized trials found a survival benefit after surgical resection combined with WBRT as compared with WBRT alone, although a third trial that included more patients with active systemic disease, did not. ${ }^{22-24}$ Because many patients have metastases in brain locations not amenable to surgical resection, the potential benefit of resection cannot be offered to all patients. In addition, if the patient is in poor medical condition, craniotomy and resection may be precluded. ${ }^{28}$

Most physicians expect a poor outcome in a patient with multiple brain metastases. Treatment regimens are often palliative in nature. Traditionally, surgical resection

Abbreviations used in this paper: $\mathrm{CI}=$ confidence interval; $\mathrm{MR}=$ magnetic resonance; $\mathrm{WBRT}=$ whole-brain radiation therapy. has been offered rarely to patients with multiple metastases, because the resection-related morbidity in multiple brain locations was believed to be excessive, and the risk for developing additional tumors was perceived to be high. ${ }^{4,28,29,31}$ Stereotactic radiosurgery, a method to deliver a single, high-dose fraction of ionizing radiation to a small, precisely defined target volume, potentially provides answers to both problems (Fig. 1). First, radiosurgery can be performed in any location in the brain, regardless of regional brain function., ${ }^{2,12}$ Second, radiosurgery can be used to treat multiple brain lesions in one setting, irrespective of tumor's histological type or configuration. ${ }^{1,10,13,14,21}$ However, because radiosurgery, like conventional surgery, is still a focused treatment, it potentially is limited by the risk of the development of additional tumors outside the initial irradiated regions. ${ }^{8}$

In the 1980s and early 1990s, it was the practice at most institutions to limit the use of radiosurgery to the management of patients with solitary tumors. This approach was more often used in patients with multiple metastases because of the high incidence of concurrent, active systemic disease. However, there was a subset of patients with multiple tumors in whom, because of the tumors' small sizes, good neurological function was maintained and who had 


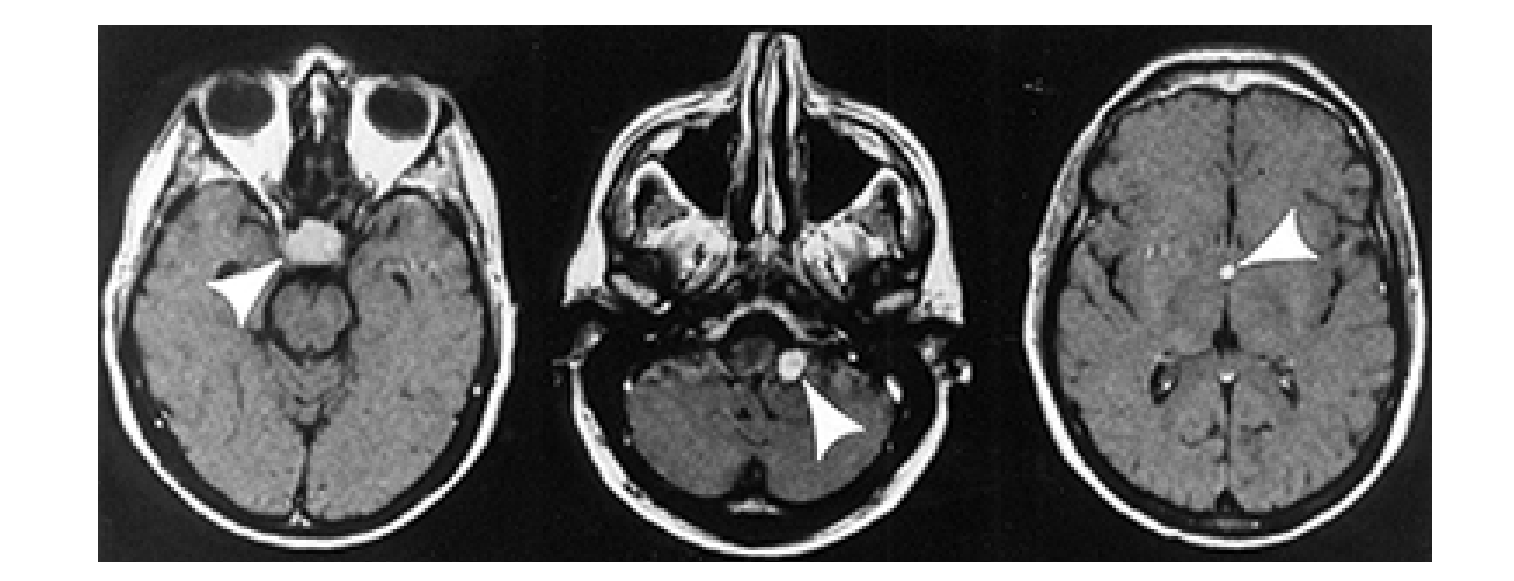

Fig. 1. Contrast-enhanced axial MR images obtained in a patient with multiple brain metastases from renal cell carcinoma (arrows). The patient refused WBRT and was one of the first patients with multiple metastases to be treated at our center. He lived 14 months and continued to practice medicine for almost 1 year after undergoing radiosurgery.

treated or controlled systemic disease. These patients usually received fractionated WBRT, up to a dose of approximately $30 \mathrm{~Gy}$. In years past, if multiple brain tumors were found after prior WBRT had been administered, then either no treatment or a smaller, fractionated whole-brain irradiation boost (10-20 Gy) was administered.

Approximately 10 years ago, we began to explore the role of radiosurgery for the treatment of patients with multiple (two to four) brain metastases (Fig. 2). Our hypothesis was that stereotactic radiosurgery in addition to WBRT would provide improved local brain tumor control and progression-free survival in patients with multiple tumors.

\section{CLINICAL MATERIAL AND METHODS}

Our randomized study was approved by the Institutional Review Board for Health Sciences at the University of Pittsburgh, and informed consent was obtained from each patient. ${ }^{18}$ Eligible patients met the following criteria: 1) histological confirmation of tumor type at the primary site or at a site of metastatic disease had been obtained in each patient; 2) all brain metastases were less than or equal to $25 \mathrm{~mm}$ in mean diameter and were located more than $5 \mathrm{~mm}$ from the optic chiasm; 3) only two, three, or four tumors were visualized on contrast-enhanced MR imaging prior to randomization; and 4) patients had a Karnofsky performance scale score less than or equal to 70 . Histological tumor types could include lung, breast, colon, renal cell, melanoma, bladder, ovarian, and uterine carcinomas. Patients were considered ineligible if they did not meet one or more of the aforementioned criteria or could not undergo MR imaging.

Local tumor control was defined as the absence of tumor growth (with caliper measurements) visualized on postradiosurgical imaging studies and no increase in clinical symptoms associated with the lesion. Our method of measuring tumor size has been previously published. ${ }^{20} \mathrm{Se}-$ rial images were interpreted by an independent, blinded observer.

At the initial examination, patient data were recorded. All patients underwent MR imaging prior to randomization. No more than four brain metastases were detected on
MR imaging in any patient prior to randomization, and all brain metastases were observed to be less than $25 \mathrm{~mm}$ in maximum diameter on neuroimaging. Systemic staging radiological studies (chest radiography, bone scanning when indicated, and computerized tomography scanning of the chest, abdomen, and pelvis as indicated) were performed within 2 months of randomization to determine active disease status. Patients were informed of the need to undergo serial MR imaging as well as clinical tests at 6 weeks and 3 months after treatment, and every 3 months thereafter.

\section{Trial Design}

Sample size calculations were based on industry standard assumptions of a power of 0.80 to detect a difference in the primary outcome measure of local control after radiosurgery combined with WBRT (expected control of $90 \%$ ) as compared with WBRT alone (expected control of $50 \%$ ). Based on these assumptions, a sample size of 44 patients was calculated for equal randomization into 22 patients per group (by coin toss). The design included a single interim analysis to be performed at $60 \%$ accrual with use of O'Brien-Fleming stopping rules. ${ }^{9,19,26}$ Statistical tests included a log-rank test for analysis of local tumor control and survival and Cox's proportional hazards model for multivariate testing. A probability value of 0.05 was used to define significance. The data were collated and reviewed by an investigator independent of each treatment arm. ${ }^{18}$

The primary outcome of this study was neuroimagingdefined control of brain disease. This primary outcome was chosen rather than survival, because the length of survival was believed to be related to extrinsic factors (other organ involvement) independent of the brain tumor therapies. Secondary outcomes included overall survival, progression-free survival, treatment-related morbidity, and the need for additional brain treatments. The primary outcome was defined by the serial MR-documented change in size and number of tumors at 1.5, 3, 6, 9, 12, 15, and 18 months following completion of either treatment. On imaging we coded the local tumor response as well as the development of new remote tumors. During follow-up ex- 


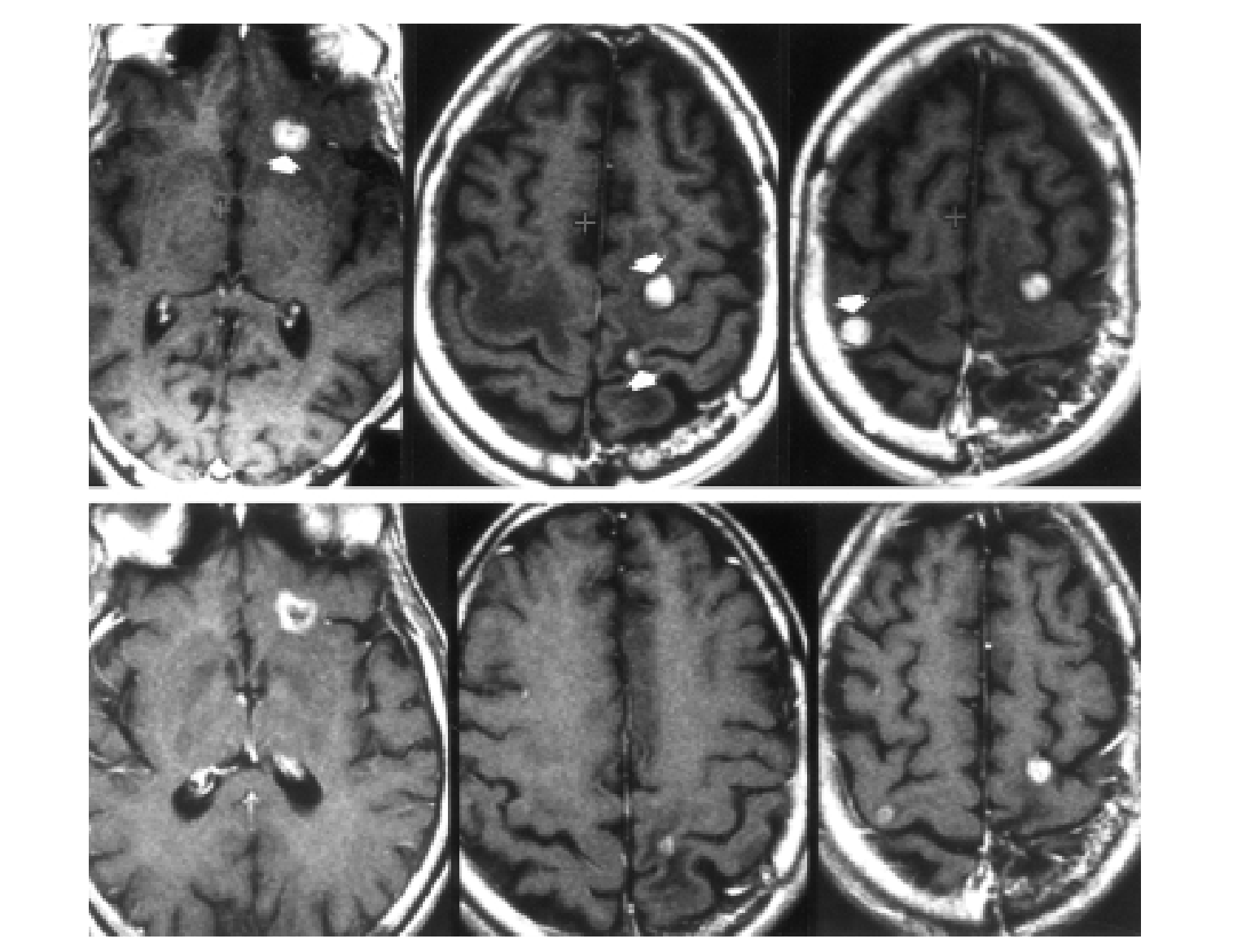

Fig. 2. Axial MR images obtained in a male patient with lung cancer metastatic to brain. He had completed WBRT following a craniotomy and tumor resection 1 year prior. Upper: Four new tumors (arrows) were identified on routine follow-up MR imaging. Lower: One year later, his brain disease remained stable, with three tumors reduced in size. He died 3 years after radiosurgery of a gastrointestinal hemorrhage.

aminations, we coded the status of non-cranial systemic disease, the requirement for other brain therapies (surgery, radiation, chemotherapy, or immunotherapy), the neurological examination, and requirement for corticosteroid therapy.

\section{Radiosurgery and Radiotherapy}

All patients randomized to treatment with fractionated WBRT were treated with megavoltage beams with a source axis distance no less than $80 \mathrm{~cm}$. Fraction sizes of 2.5 Gy were used. A midplane dose of $30 \mathrm{~Gy}$ in 12 fractions was delivered. Radiosurgery could precede, follow, or be performed within the time course of WBRT. The maximum time interval between WBRT and radiosurgery in patients randomized to radiosurgery was 1 month.

Patients randomized to the WBRT/radiosurgery group underwent gamma knife radiosurgery (Elekta Instruments, Atlanta, GA) administered using stereotactic MR guidance. Dose planning was performed using an imageintegration on a computer workstation. All known tumors were irradiated. The $50 \%$ or greater isodose (16 Gy) was used to irradiate the tumor margin in all patients (Fig. 3). This tumor margin radiosurgery dose in patients who had and had not undergone prior radiotherapy was selected so that the predicted biological effect on normal brain tissue and risk of complications (approximately 3\%) would be the same. ${ }^{11}$ Patients with symptomatic cerebral edema were treated with oral corticosteroid medications (dexamethasone $24 \mathrm{mg}$ /day in divided doses or less).

\section{RESULTS}

Twenty-seven patients were randomized as part of the initial management plan after the diagnosis of multiple brain metastases had been confirmed. A histological diagnosis of recent cancer was made in all patients. The neuroimaging findings were characteristic of brain metastases in all patients (contrast-enhanced mass visualized on short TR images surrounded by a region of high signal on long TR images). The characteristics of the patients are listed in Table 1. Fourteen patients were randomized to receive WBRT as initial management. Thirteen patients were randomized to WBRT combined with stereotactic radiosurgery. The groups were well matched according to age (mean age 58 and 59 years, respectively), sex, Karnofsky performance scale score (median score 100 in both groups), and distribution of tumor histological types. Nonsmall cell lung cancer was the most frequent diagnosis in each group, and similar proportions of melanoma, renal cell carcinoma, and breast carcinoma were present in each 


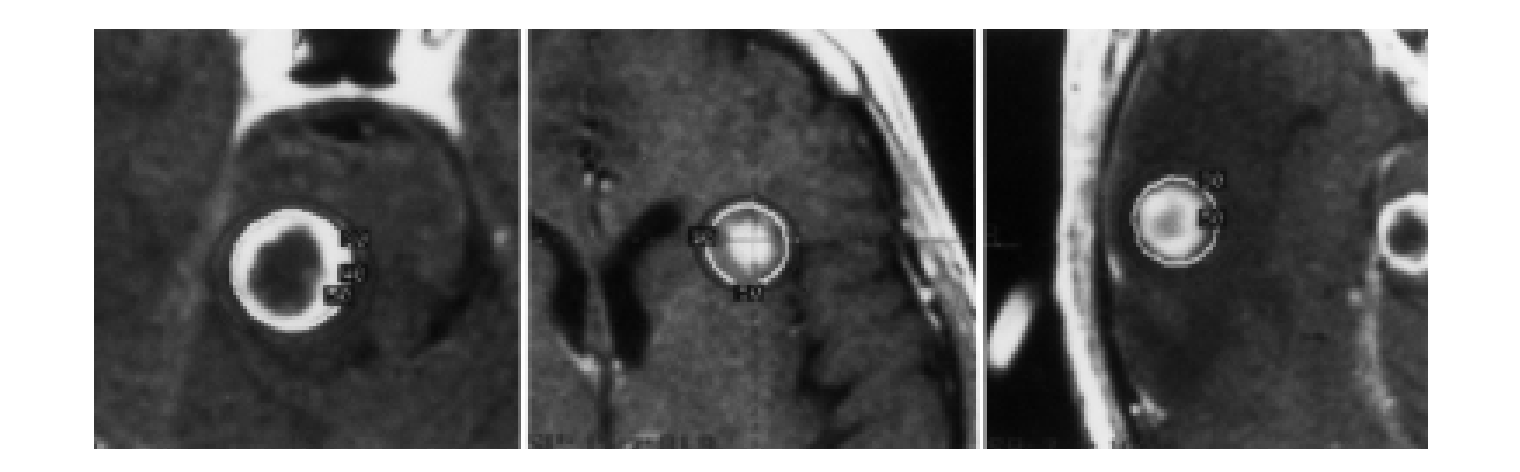

Fig. 3. Axial MR images obtained at the time of radiosurgery in a patient with three metastases from renal cell carcinoma. The tumors were irradiated in the midbrain, left frontal lobe, and right temporal lobe, following WBRT. The 50\% isodose line was used to cover the tumor margin.

group. Overall, in $71 \%$ of the WBRT-alone group active systemic disease was found, and that in patients receiving WBRT/radiosurgery was $62 \%$. Finally, the distribution in the number of brain metastases was similar between groups. Most patients (79\% in the WBRT group and 62\% in the WBRT/radiosurgery group) harbored two tumors.

The trial was stopped following an interim analysis after 27 patients had been entered into the study. ${ }^{18}$ At this point a log-rank analysis revealed significant benefit in the rate of local tumor control after radiosurgery/WBRT $(\mathrm{p}=$ $0.0016)$. The level of significance exceeded the nominal significance value of $p=0.0092$ required to stop the trial in accordance with the O'Brien-Fleming stopping rules stated at inception. Because patient accrual was slow and based on the findings of the interim analysis, the investigators believed it inappropriate to withhold therapy shown to be statistically efficacious and safe.

We found a significant improvement in the local tumor control rate after WBRT/radiosurgery as compared with WBRT alone. The rate of local failure at 1 year was $100 \%$ after WBRT alone (this included patients who died before

TABLE 1

Clinical characteristics in patients with multiple brain metastases randomized to two treatment groups

\begin{tabular}{lcc}
\hline \hline Characteristic & $\begin{array}{c}\text { WBRT Group } \\
(14 \text { patients })\end{array}$ & $\begin{array}{c}\text { WBRT/Radio- } \\
\text { surgery Group } \\
(13 \text { patients })\end{array}$ \\
\hline mean age (range) & $58(33-77)$ & $59(46-74)$ \\
male/female ratio & $7 / 7$ & $9 / 4$ \\
tumor histological type & & \\
lung carcinoma & 7 & 5 \\
melanoma & 3 & 2 \\
renal cell carcinoma & 2 & 2 \\
breast carcinoma & 2 & 2 \\
other carcinoma & 0 & 8 \\
systemic disease & $10(71 \%)$ & 5 \\
none (other than brain) & 4 & 4 \\
present but not lung & 5 & 4 \\
present including lung & 5 & 8 \\
no. of tumors & & 3 \\
two & 11 & 2 \\
three & 1 & \\
four & 2 & \\
\hline
\end{tabular}

1 year in addition to those still living, in all of whom tumor progression was demonstrated); however, this rate was only $8 \%$ at 1 year in surviving patients who underwent boost radiosurgery. The median time to local failure was 6 months after WBRT alone (95\% CI 3.5-8.5) as compared with 36 months (95\% CI 15.6-57) after WBRT/ radiosurgery $(\mathrm{p}=0.0005)$. The median time to the manifestation of any brain failure (progression of the initial tumors or the development of new tumors) was 5 months (95\% CI 3.2-6.8) after WBRT alone and 34 months after WBRT/radiosurgery $(\mathrm{p}=0.002)$. Local tumor control was not dependent on tumor histological type (breast versus lung/other versus melanoma/renal cell cancer; $\mathrm{p}=0.85$ ). Progression of brain disease did not correlate with the number of initially detected brain metastases $(p=0.25)$. The presence of active systemic disease outside the brain also did not correlate with brain control $(\mathrm{p}=0.26){ }^{18}$

No difference in overall survival was noted between the two groups based on the patients' initial brain tumor management, although there was a trend toward longer survival in the group receiving the combined therapies. Patients who received WBRT alone at diagnosis lived a median of 7.5 months (95\% CI 4.6-10.4), whereas those who received WBRT/radiosurgery lived 11 months $(95 \%$ CI 3.8-18.2; $\mathrm{p}=0.22$ ). Some patients in the WBRT-alone group who developed later progression of brain disease (local or remote) underwent salvage radiosurgery at that time (patients with marked systemic disease progression received no further treatment). In an analysis of the stratification of the WBRT-alone group we found that patients who received only WBRT and no later radiosurgery lived a median of 7 months (95\% CI 3.6-10.4), whereas those who underwent delayed radiosurgery lived a median of 11 months (95\% CI 7.1-14.9; and this was similar to the outcome found in those randomized to the combined treatment group). A difference in survival was found between these three groups (WBRT alone, WBRT and delayed salvage radiosurgery, and WBRT/initial radiosurgery; $p=$ $0.04)$. In a strict analysis of patients who received WBRT alone and no further salvage brain management as compared with those who received WBRT/initial radiosurgery we identified a survival improvement in those undergoing radiosurgery $(p=0.028)$. Patients who received any additional form of brain management (radiosurgery, surgical resection (one case), or additional whole-brain irradiation 
but not chemotherapy) also lived longer (median survival 11 months, 95\% CI 6.7-15.3) than those patients who did not (median survival 5.5 months, 95\% CI 3.3-7.7; $\mathrm{p}=0.027) .^{18}$

The extent and type of systemic disease proved to be important. When outcome data in patients from both treatment arms were combined, median survival was 14 months (95\% CI 12.7-15.3) in those with no systemic disease (other than their primary tumor and brain tumors), 7.5 months (95\% CI 6.0-8.9) in those with systemic disease excluding lung metastases, and 5.5 months $(95 \%$ CI 3.5-7.5) in those with systemic disease that included lung metastases $(\mathrm{p}=0.02)$. The importance of additional systemic disease as a negative predictor for survival was important even without separating out patients with lung metastases $(\mathrm{p}=0.05){ }^{18}$

\section{DISCUSSION}

\section{Expectations for Patients With Multiple Brain Metastases}

The diagnosis of multiple brain metastases in a cancer patient is frequently made and has been associated with a dismal prognosis. The results of our randomized study indicate that such an outcome may not be inevitable, as progression-free survival is a real possibility for patients with two, three, or four brain tumors, regardless of the tumor's histological type. Management of the brain tumors with WBRT and stereotactic radiosurgery leads to a high rate of local brain disease control which, in turn, places the onus on continued care of extracranial metastatic disease to improve survival length further.

\section{The Role of Resection}

The addition of surgical resection to WBRT appeared to double survival lengths in several randomized trials, to the range of 10 to 12 months. ${ }^{24,28,30}$ These data were obtained only in patients with single brain metastases. The importance of systemic disease has again been correlated with survival. ${ }^{6,15,23,28,30}$ In a recent randomized trial reported by Mintz, et al., ${ }^{22}$ the authors found no survival benefit to resection combined with WBRT as compared with WBRT alone in patients with single tumors, perhaps reflecting a higher incidence of active systemic disease than that seen in other randomized trials.

In two recent studies the authors have expanded the scope of surgical resection to the treatment of patients with multiple brain metastases. In a retrospective review, Bindal, et al., ${ }^{3}$ reported a 14-month average survival after the complete removal of multiple tumors in 26 cases, which matched the 14-month survival observed in a control group with single tumors matched for tumor type, time from presentation to metastasis, and extent of systemic disease. After incomplete resection (30 cases), survival time was no longer than that conferred by radiation treatment alone (average approximately 6 months). Hazuka, et al., ${ }^{17}$ found only a 5-month average survival length in patients who underwent with resection of multiple metastases (18 cases), but only in one of their patients was total resection of all metastases achieved, and that patient lived 48 months. One important finding common to all of these studies was that the initial diagnosis of mul- tiple brain metastases was not necessarily followed by the finding of an increasing number of brain tumors over the ensuing months. We believe that surgical resection remains important for the removal of a large symptomatic metastasis. Such surgical intervention, if successful, would allow the patient to undergo other treatments for the remaining tumors. The primary benefit of radiosurgery over resection in patients with multiple metastases is that it avoids the need for multiple craniotomies.

\section{The Treatment of Miliary Metastases}

Some patients are found to harbor many small brain metastases, often from 10 to 100 such tumors. In these patients, the prognosis is guarded but depends again, to a large degree, on the extent of extracranial disease. When no other treatment options were available, we have performed radiosurgery in a small group of young patients, in each of whom there were fewer than 12 tumors. Surgeons at some centers apparently have performed radiosurgery in patients with upward of 100 tumors. We believe that this approach is unlikely to provide the patient with any worthwhile benefit.

\section{Treatment Goals}

To provide effective palliation, radiosurgery combined with radiation therapy must achieve several goals. First, the treated tumors must not increase in size or cause additional neurological symptoms. Second, these benefits should be available to patients regardless of tumor histological type. In our randomized trial, no patient developed additional neurological symptoms from the treated tumors. New symptoms did develop from new tumors that were diagnosed later on, and these patients received additional brain treatment (usually radiosurgery) if the burden of their systemic disease was not out of control.

Achieving a high rate of local disease control can be enhanced by increasing the delivered radiation dose. Shiau, et al., ${ }^{27}$ increased the 1 -year control rate from 77 to $90 \%$ when a minimum dose of greater than or equal to 18 Gy was delivered to the tumor. Histological studies of metastases after radiosurgery have shown reduced growth potential with molecular analyses. ${ }^{16}$

\section{CONCLUSIONS}

As improvements in the care of cancer patients are achieved, longer survival times will be expected. The diagnosis of a patient with multiple brain metastases will become even more common. The combination of WBRT and stereotactic radiosurgery for the treatment of patients with two to four tumors significantly improves the control of brain disease. Although we found no evidence of improved survival in the randomized patients, those randomized to WBRT-alone group who were then selected to receive later salvage radiosurgery lived longer than those who were not selected to undergo that procedure. Wholebrain radiation therapy alone does not appear to provide lasting and effective care for most patients. For patients with two to four brain metastases less than $25 \mathrm{~mm}$ in diameter who have no other extracranial disease other than their primary tumor or stable extracranial disease, radiosurgery combined with WBRT radiation therapy is our 
preferred management option. Such an approach is particularly important in patients in whom a good prognosis is expected. In future trials we plan to explore whether WBRT will benefit patients with one or two tumors. Vigilant management of extracranial cancer should improve survival length further.

\section{References}

1. Adler JR, Cox RS, Kaplan I, et al: Stereotactic radiosurgical treatment of brain metastases. J Neurosurg 76:444-449, 1992

2. Auchter RM, Lamond JP, Alexander E, et al: A multiinstitutional outcome and prognostic factor analysis of radiosurgery for resectable single brain metastasis. Int J Radiat Oncol Biol Phys 35:27-35, 1996

3. Bindal RK, Sawaya R, Leavens ME, et al: Surgical treatment of multiple brain metastases. J Neurosurg 79:210-216, 1993

4. Black P: Brain metastasis: current status and recommended guidelines for management. Neurosurgery 5:617-631, 1979

5. Borgelt B, Gelber R, Kramer S, et al: The palliation of brain metastases: final results of the first two studies by the Radiation Therapy Oncology Group. Int J Radiat Oncol Biol Phys 6: $1-9,1980$

6. Buatti JM, Friedman WA, Bova FJ, et al: Treatment selection factors for stereotactic radiosurgery of intracranial metastases. Int J Radiat Oncol Biol Phys 32:1161-1166, 1995

7. Cairncross JG, Kim JH, Posner JB: Radiation therapy for brain metastases. Ann Neurol 7:529-541, 1980

8. Deutsch M, Parsons JA, Mercado R Jr: Radiotherapy for intracranial metastases. Cancer 34:1607-1611, 1974

9. Emerson SS, Fleming TR: Interim analyses in clinical trials. Oncology 4:126-134, 136, 1990

10. Engenhart R, Kimmig BN, Hover KH, et al: Long term followup for brain metastases treated by percutaneous stereotactic single high-dose irradiation. Cancer 71:1353-1361, 1993

11. Flickinger JC: An integrated logistic formula and prediction of complications from radiosurgery. Int J Radiat Oncol Biol Phys 17:879-885, 1989

12. Flickinger JC, Kondziolka D, Lunsford LD, et al: A multi-institutional experience with stereotactic radiosurgery for solitary brain metastasis. Int J Radiat Oncol Biol Phys 28:797-802, 1994

13. Flickinger JC, Lunsford LD, Somaza S, et al: Radiosurgery: its role in brain metastasis management. Neurosurg Clin North Am 7:497-504, 1996

14. Fuller BG, Kaplan ID, Adler J, et al: Stereotaxic radiosurgery for brain metastases: the importance of adjuvant whole brain irradiation. Int J Radiat Oncol Biol Phys 23:413-418, 1992

15. Galicich JH, Sundaresan N, Arbit E, et al: Surgical treatment of single brain metastasis: factors associated with survival. Cancer 5:381-386, 1980

16. Harris OA, Adler JR: Analysis of the proliferative potential of residual tumor after radiosurgery for intraparenchymal brain metastases. J Neurosurg 85:667-671, 1996

17. Hazuka MB, Burleson WD, Stroud DN, et al: Multiple brain metastases are associated with poor survival in patients treated with surgery and radiotherapy. J Clin Oncol 11:369-373, 1993

18. Kondziolka D, Patel A, Lunsford LD, et al: Stereotactic radiosurgery plus whole brain radiotherapy versus radiotherapy alone for patients with multiple brain metastases. Int J Radiat Oncol Biol Phys 45:427-434, 1999

19. Lewis RJ: An introduction to the use of interim data analysis in clinical trials. Ann Emerg Med 22:1463-1469, 1993

20. Linskey M, Lunsford LD, Flickinger JC: Neuroimaging of nerve sheath acoustic tumors after stereotaxic radiosurgery. AJNR 12:1165-1175, 1991

21. Loeffler JS, Kooy HM, Wen PY, et al: The treatment of recurrent brain metastases with stereotactic radiosurgery. J Clin Oncol 8:576-582, 1990

22. Mintz AH, Kestle J, Rathbone MP, et al: A randomized trial to assess the efficacy of surgery in addition to radiotherapy in patients with a single cerebral metastases. Cancer 78:1470-1476, 1996

23. Noordijk EM, Vecht CJ, Haaxma-Reiche H, et al: The choice of treatment of single brain metastasis should be based on extracranial tumor activity and age. Int J Radiat Oncol Biol Phys 29:711-717, 1994

24. Patchell RA, Tibbs PA, Walsh JW, et al: A randomized trial of surgery in the treatment of single metastases to the brain. $\mathbf{N}$ Engl J Med 322:494-500, 1990

25. Pickren JW, Lopez G, Tzukada Y, et al: Brain metastases: an autopsy study. Cancer Treatment Symptoms 2:295-313, 1983

26. Pignon JP, Arriagada R: Early stopping rules and long-term follow-up in phase III trials. Lung Cancer 10 (Suppl 1): S151-S159, 1994

27. Shiau CY, Sneed PK, Shu HK, et al: Radiosurgery for brain metastases: relationship of dose and pattern of enhancement to local control. Int J Radiat Oncol Biol Phys 37:375-383, 1997

28. Smalley SR, Laws ER Jr, O'Fallon JR, et al: Resection for solitary brain metastasis. Role of adjuvant radiation and prognostic variables in 229 patients. J Neurosurg 77:531-540, 1992

29. Sundaresan N, Galicich JH, Beattie EJ Jr: Surgical treatment of brain metastases from lung cancer. J Neurosurg 58:666-671, 1983

30. Vecht CJ, Haaxma-Reiche H, Noordijk EM, et al: Treatment of single brain metastasis: radiotherapy alone or combined with neurosurgery? Ann Neurol 33:583-590, 1993

31. Young RF, Jacques DB, Duma C, et al: Gamma knife radiosurgery for treatment of multiple brain metastases. A comparison of patients with single versus multiple lesions. Radiosurgery 1:92-101, 1995

Manuscript received June 20, 2000.

Accepted in final form July 10, 2000.

Dr. Kondziolka was supported by National Institutes of Health Grant No. K08 NS01723.

Address reprint requests to: Douglas Kondziolka, M.D., UPMCPresbyterian, Department of Neurological Surgery, Suite B-400, 200 Lothrop Street, Pittsburgh, Pennsylvania, 15213. 